\title{
Model Reduction of Fuzzy Logic Systems
}

\author{
Zhandong Yu, ${ }^{1}$ Jinyong $Y u^{2}$ and Hamid Reza Karimi ${ }^{1,3}$ \\ ${ }^{1}$ Research Institute of Mechatronics and Automation, Bohai University, Jinzhou, Liaoning 121013, China \\ ${ }^{2}$ Department of Control Science and Engineering, Harbin Institute of Technology, Harbin 150080, China \\ ${ }^{3}$ Department of Engineering, the Faculty of Engineering and Science, University of Agder, 4898 Grimstad, Norway
}

Correspondence should be addressed to Zhandong Yu; yuzhandong2014@gmail.com

Received 25 March 2014; Accepted 20 April 2014; Published 11 May 2014

Academic Editor: M. Chadli

Copyright (C) 2014 Zhandong Yu et al. This is an open access article distributed under the Creative Commons Attribution License, which permits unrestricted use, distribution, and reproduction in any medium, provided the original work is properly cited.

\begin{abstract}
This paper deals with the problem of $\mathscr{L}_{2}-\mathscr{L}_{\infty}$ model reduction for continuous-time nonlinear uncertain systems. The approach of the construction of a reduced-order model is presented for high-order nonlinear uncertain systems described by the T-S fuzzy systems, which not only approximates the original high-order system well with an $\mathscr{L}_{2}-\mathscr{L}_{\infty}$ error performance level $\gamma$ but also translates it into a linear lower-dimensional system. Then, the model approximation is converted into a convex optimization problem by using a linearization procedure. Finally, a numerical example is presented to show the effectiveness of the proposed method.
\end{abstract}

\section{Introduction}

It is well known that fuzzy logic theory is an effective approach for the manipulation of complex nonlinear systems. Recently, it has witnessed the rapidly growing popularity of fuzzy control systems in practical applications. Among various kinds of fuzzy models, the Takagi-Sugeno (T-S) fuzzy model [1] is viewed as one of the most popular models. A number of IF-THEN rules, which describe the local input-output relationships of original nonlinear system, are introduced to this kind of model. The weighted sum of all local linear systems have been obtained by constructing a group of weight functions from the membership functions in each rule. This weighted sum is equivalent to the original nonlinear system, which can be regarded as a special kind of time-varying linear system. Thus, most of the classical linear control methods will be applicable. There are numerous cases in this approach. For example, stability analysis was investigated in [2-8], the controller design was presented in [9-18], state estimation and filtering problems were addressed in [19-23], and fault detection problem was widely studied in [24-26].

On the other hand, since high-dimensional complex mathematical modeling of physical systems and processes in many areas of engineering is frequently encountered, the problem of model reduction has obtained a considerable attention. This model brings severe hardship to analyze and synthesize the concerning systems. Therefore, it is desirable to find a possible lower-order model to approximate the original one without introducing significant performance error in real-word applications. There are many important reported results in the past decades. These have been developed to settle the difficulty of model approximation, such as the $\mathscr{L}_{2}$ $\mathscr{L}_{\infty}$ approach [27, 28], the $\mathscr{H}_{\infty}$ (or equivalently, energyto-energy) approach [29-32], the $\mathscr{H}_{2}$ approach [33, 34], and the Hankel-norm approach [35]. Very recently, as the rapid developments of the linear matrix inequality [36-38] technique, this approach has been explored to settle the model reduction problem for different classes of systems in effect, involving time-delay systems [28], switched hybrid systems [30], and Markovian jump systems [39]. However, to the best of the authors' knowledge, few results on $\mathscr{L}_{2}-\mathscr{L}_{\infty}$ model reduction for nonlinear uncertain systems described in the T-S fuzzy framework are available, and it still remains challenging. Thus, research in this area should be of both theoretical and practical importance, which motivates us to carry out the present work. 
In this work, the $\mathscr{L}_{2}-\mathscr{L}_{\infty}$ model simplification problem is investigated for a class of nonlinear uncertain systems in the T-S fuzzy framework. For a given stable T-S fuzzy uncertain system, our attention is focused on the construction of reduced-order model, which approximates the original high-order system well in an $\mathscr{L}_{2}-\mathscr{L}_{\infty}$ error performance level $\gamma$. The rest of this paper is organized as follows. The problem formulation and some preliminaries are presented in Section 2. $\mathscr{L}_{2}-\mathscr{L}_{\infty}$ performance analysis and $\mathscr{L}_{2}-\mathscr{L}_{\infty}$ model reduction are given in Sections 3 and 4, respectively. A numerical example is provided in Section 5 and we conclude this paper in Section 6.

Notations. " $T$ " stands for matrix transposition; $\mathbb{R}^{n}$ denotes the $n$-dimensional Euclidean space; the notation $P>0$ means that $P$ is real symmetric and positive definite; $I$ and 0 represent the identity matrix and a zero matrix, respectively; $\operatorname{diag}(. .$.$) stands for a block-diagonal matrix; \|\cdot\|$ denotes the Euclidean norm of a vector and its induced norm of a matrix; and the signals that are square integrable over $[0, \infty)$ are denoted by $\mathscr{L}_{2}[0, \infty)$ with the norm $\|\cdot\|_{2}$. In symmetric block matrices, we use an asterisk $(*)$ to represent a term that is induced by symmetry. Matrices, if their dimensions are not explicitly stated, are assumed to be compatible for algebraic operations.

\section{Problem Formulation and Preliminaries}

In this paper, we consider a class of uncertain nonlinear systems which can be described by the following T-S fuzzy uncertain model.

\subsection{Plant Form}

Rule i. IF $\theta_{1}(t)$ is $\mu_{i 1}$ and $\theta_{2}(t)$ is $\mu_{i 2}$ and $\ldots$ and $\theta_{p}(t)$ is $\mu_{i p}$, then

$$
\begin{gathered}
\dot{x}(t)=\left[A_{i}+\Delta A_{i}(t)\right] x(t)+\left[B_{i}+\Delta B_{i}(t)\right] u(t), \\
y(t)=C_{i} x(t), \quad \forall t, \quad i=1,2, \ldots, r,
\end{gathered}
$$

where $\mu_{i 1}, \ldots, \mu_{i p}$ are the fuzzy sets; $x(t) \in \mathbb{R}^{n}$ is the state vector; $u(t) \in \mathbb{R}^{m}$ is the input which belongs to $\mathscr{L}_{2}[0, \infty) ; y(t) \in \mathbb{R}^{p}$ is the output; $\left(A_{i}, B_{i}, C_{i}\right)$ are matrices of compatible dimensions, $r$ is the number of IF-THEN rules, $\theta_{1}(t), \theta_{2}(t), \ldots, \theta_{p}(t)$ are the premise variables; $\Delta A_{i}(t)$ and $\Delta B_{i}(t)$ are time-varying matrices with appropriate dimensions, which are defined as follows:

$$
\begin{array}{r}
\Delta A_{i}(t)=U_{i} F(t) M_{i}, \quad \Delta B_{i}(t)=U_{i} F(t) N_{i}, \\
\forall t, i=1, \ldots, r,
\end{array}
$$

where $U_{i}, V_{i}, M_{i}$, and $N_{i}$ are known constant real matrices of appropriate dimensions and $F(t)$ is an unknown matrix function with Lebesgue-measurable elements and satisfies

$$
F(t)^{T} F(t) \leqslant I, \quad \forall t, i=1, \ldots, r .
$$

It is assumed that the premise variables do not depend on the input variables $u(t)$. Given a pair of $(x(t), u(t))$, the output of the T-S fuzzy systems with uncertainty is inferred as follows:

$$
\begin{array}{r}
\dot{x}(t)=\sum_{i=1}^{r} h_{i}(\theta(t))\left\{\left[A_{i}+\Delta A_{i}(t)\right] x(t)\right. \\
\left.+\left[B_{i}+\Delta B_{i}(t)\right] u(t)\right\}, \\
y(t)=\sum_{i=1}^{r} h_{i}(\theta(t)) C_{i} x(t), \quad \forall t,
\end{array}
$$

with

$$
h_{i}(\theta(t))=\frac{v_{i}(\theta(t))}{\sum_{i=1}^{r} v_{i}(\theta(t))}, \quad v_{i}(\theta(t))=\prod_{j=1}^{p} \mu_{i j}\left(\theta_{j}(t)\right),
$$

where $\mu_{i j}\left(\theta_{j}(t)\right)$ is the grade of membership of $\theta_{j}(t)$ in $\mu_{i j}$. Suppose that $v_{i}(\theta(t)) \geq 0, i=1,2, \ldots, r, \sum_{i=1}^{r} v_{i}(\theta(t))>0$ for all $t$. Therefore, $h_{i}(\theta(t)) \geq 0$ for $i=1,2, \ldots, r$ and $\sum_{i=1}^{r} h_{i}(\theta(t))=1$ for all $t$.

Here, the system in (4) is approximated by a reducedorder uncertain system described by

$$
\begin{gathered}
\dot{\hat{x}}(t)=[\widehat{A}+\Delta \widehat{A}(t)] \widehat{x}(t)+[\widehat{B}+\Delta \widehat{B}(t)] u(t), \\
\widehat{y}(t)=\widehat{C} \hat{x}(t), \quad \forall t,
\end{gathered}
$$

where $\widehat{x}(t) \in \mathbb{R}^{k}$ is the state vector of the reduced-order system $(\widehat{\Sigma})$ with $k<n$; it is assumed that $\Delta \widehat{A}(t)$ and $\Delta \widehat{B}(t)$ are time-varying matrices and have the structure of

$$
\Delta \widehat{A}(t)=\widehat{U} F(t) \widehat{M}, \quad \Delta \widehat{B}(t)=\widehat{U} F(t) \widehat{N}, \quad \forall t
$$

with $F(k)$ being feasible. This assumption means that the uncertainties in both the original models and reduced order models should come from the same sources but with the different weighting matrices $\left\{U_{i}, M_{i}, N_{i}\right\}$ and $\{\widehat{U}, \widehat{M}, \widehat{N}\} . \widehat{A}, \widehat{B}$, $\widehat{C}, \widehat{U}, \widehat{M}$, and $\widehat{N}$ are appropriately dimensioned matrices to be determined later.

Augmenting the model in (4) to include the states in (6), we obtain the following error system as

$$
\begin{array}{r}
\dot{\xi}(t)=\sum_{i=1}^{r} h_{i}(\theta(t))\left\{\left[\widetilde{A}_{i}+\Delta \widetilde{A}_{i}(t)\right] \xi(t)\right. \\
\left.+\left[\widetilde{B}_{i}+\Delta \widetilde{B}_{i}(t)\right] u(t)\right\}, \\
e(t)=\sum_{i=1}^{r} h_{i}(\theta(t)) \widetilde{C}_{i} \xi(t), \quad \forall t,
\end{array}
$$


where $\xi(t) \triangleq\left[\begin{array}{ll}x^{T}(t) & \widehat{x}^{T}(t)\end{array}\right]^{T}, e(t) \triangleq y(t)-\widehat{y}(t)$, and

$$
\begin{array}{ll}
\widetilde{A}_{i} \triangleq\left[\begin{array}{cc}
A_{i} & 0 \\
0 & \widehat{A}
\end{array}\right], \quad \Delta \widetilde{A}_{i}(t) \triangleq\left[\begin{array}{cc}
\Delta A_{i}(t) & 0 \\
0 & \Delta \widehat{A}(t)
\end{array}\right], \\
\widetilde{C}_{i} \triangleq\left[\begin{array}{c}
C_{i}^{T} \\
-\widetilde{C}^{T}
\end{array}\right], \quad \widetilde{B}_{i} \triangleq\left[\begin{array}{c}
B_{i} \\
\widehat{B}
\end{array}\right], \quad \Delta \widetilde{B}_{i}(t) \triangleq\left[\begin{array}{c}
\Delta B_{i}(t) \\
\Delta \widehat{B}(t)
\end{array}\right] .
\end{array}
$$

Define

$$
\begin{array}{r}
\Delta \widetilde{A}_{i}(t)=\widetilde{U}_{i} \widetilde{F}(t) \widetilde{M}_{i}, \quad \Delta \widetilde{B}_{i}(t)=\widetilde{U}_{i} \widetilde{F}(t) \widetilde{N}_{i}, \\
\forall t, i=1, \ldots, r,
\end{array}
$$

where

$$
\begin{aligned}
& \widetilde{U}_{i} \triangleq\left[\begin{array}{cc}
U_{i} & 0 \\
0 & \widehat{U}
\end{array}\right], \quad \widetilde{N}_{i} \triangleq\left[\begin{array}{c}
N_{i} \\
\widehat{N}
\end{array}\right] \\
& \widetilde{F}(t) \triangleq\left[\begin{array}{cc}
F(t) & 0 \\
0 & F(t)
\end{array}\right], \quad \widetilde{M}_{i} \triangleq\left[\begin{array}{cc}
M_{i} & 0 \\
0 & \widehat{M}
\end{array}\right] .
\end{aligned}
$$

Before proceeding, we introduce the following definitions, which will be of help in deriving our main results in the sequel.

Definition 1. The equilibrium $\xi^{*}=0$ of T-S fuzzy uncertain system in (8) with $u(t)=0$ is asymptotically stable if its solution $\xi(t)$ satisfies

$$
\lim _{t \rightarrow \infty}\|\xi(t)\|^{2}=0
$$

Definition 2. Given a scalar $\gamma>0$, the T-S fuzzy uncertain system in (8) is asymptotically stable with an $\mathscr{L}_{2}-\mathscr{L}_{\infty}$ performance level $\gamma$ if it is asymptotically stable when $u(t) \equiv 0$ and, under zero initial condition and for all nonzero $u(t) \epsilon$ $\mathscr{L}_{2}[0, \infty)$, the following holds:

$$
\sup _{\forall t}\left[e^{T}(t) e(t)\right]<\gamma^{2} \int_{0}^{\infty} u^{T}(t) u(t) d t
$$

Therefore, the $\mathscr{L}_{2}-\mathscr{L}_{\infty}$ model reduction problem addressed in this paper can be formulated as follows: given the high-order T-S fuzzy uncertain system in (4) and a scalar $\gamma>0$, determine a reduced-order uncertain model in (6) such that the resulting error system in (8) is asymptotically stable with an $\mathscr{L}_{2}-\mathscr{L}_{\infty}$ performance level $\gamma$.

\section{3. $\mathscr{L}_{2}-\mathscr{L}_{\infty}$ Performance Analysis}

Firstly, we investigate the asymptotic stability and $\mathscr{L}_{2}-\mathscr{L}_{\infty}$ performance of the error system in (8) and have the following result.
Theorem 3. Given a scalar $\gamma>0$, the error system in (8) is asymptotically stable with an $\mathscr{L}_{2}-\mathscr{L}_{\infty}$ performance level $\gamma$ if there exists matrix $P>0$ such that for $i=1,2, \ldots, r$,

$$
\left[\begin{array}{ccccc}
P \widetilde{A}_{i}+\widetilde{A}_{i}^{T} P & P \widetilde{B}_{i} & P \widetilde{U}_{i} & \widetilde{M}_{i}^{T} & 0 \\
\star & -I & 0 & 0 & \widetilde{N}_{i}^{T} \\
\star & \star & -\frac{\mu}{2} I & 0 & 0 \\
\star & \star & \star & -\frac{1}{\mu} I & 0 \\
\star & \star & \star & \star & -\frac{1}{\mu} I
\end{array}\right]<0,
$$

Proof. Choose a Lyapunov function as

$$
V(\xi(t), t) \triangleq \xi^{T}(t) P \xi(t), \quad P>0 .
$$

Along the trajectories of the error system in (8), and considering the differential of the Lyapunov function in (16), we have

$\dot{V}(\xi(t), t)$

$$
\begin{aligned}
& =\sum_{i=1}^{r} h_{i}(\theta(t))\left\{\left[\widetilde{A}_{i}+\Delta \widetilde{A}_{i}(t)\right] \xi(t)+\left[\widetilde{B}_{i}+\Delta \widetilde{B}_{i}(t)\right] u(t)\right\}^{T} P \xi(t) \\
& +\sum_{i=1}^{r} h_{i}(\theta(t)) \xi^{T}(t) P\left\{\left[\widetilde{A}_{i}+\Delta \widetilde{A}_{i}(t)\right] \xi(t)\right. \\
& \left.+\left[\widetilde{B}_{i}+\Delta \widetilde{B}_{i}(t)\right] u(t)\right\} \\
& =\sum_{i=1}^{r} h_{i}(\theta(t)) \xi^{T}(t)\left\{P\left[\widetilde{A}_{i}+\Delta \widetilde{A}_{i}(t)\right]\right. \\
& \left.+\left[\widetilde{A}_{i}+\Delta \widetilde{A}_{i}(t)\right]^{T} P\right\} \xi(t) \\
& +2 \sum_{i=1}^{r} h_{i}(\theta(t)) \xi^{T}(t) P\left[\widetilde{B}_{i}+\Delta \widetilde{B}_{i}(t)\right] u(t), \\
& =\sum_{i=1}^{r} h_{i}(\theta(t)) \xi^{T}(t)\left\{P\left[\widetilde{A}_{i}+\widetilde{U}_{i} \widetilde{F}(t) \widetilde{M}_{i}\right]\right. \\
& \left.+\left[\widetilde{A}_{i}+\widetilde{U}_{i} \widetilde{F}(t) \widetilde{M}_{i}\right]^{T} P\right\} \xi(t) \\
& +2 \sum_{i=1}^{r} h_{i}(\theta(t)) \xi^{T}(t) P\left[\widetilde{B}_{i}+\widetilde{U}_{i} \widetilde{F}(t) \widetilde{N}_{i}\right] u(t) .
\end{aligned}
$$


Therefore, when assuming the zero input, that is, $u(t)=0$, we have from (17) that

$$
\begin{aligned}
& \dot{V}(\xi(t), t) \\
& =\sum_{i=1}^{r} h_{i}(\theta(t)) \xi^{T}(t)\left\{P\left[\widetilde{A}_{i}+\Delta \widetilde{A}_{i}(t)\right]\right. \\
& \left.+\left[\widetilde{A}_{i}+\Delta \widetilde{A}_{i}(t)\right]^{T} P\right\} \xi(t) \\
& =\sum_{i=1}^{r} h_{i}(\theta(t)) \xi^{T}(t)\left[P \widetilde{A}_{i}+\widetilde{A}_{i}^{T} P+P \Delta \widetilde{A}_{i}(t)+\Delta \widetilde{A}_{i}(t)^{T} P\right] \xi(t) \\
& =\sum_{i=1}^{r} h_{i}(\theta(t)) \xi^{T}(t)\left[P \widetilde{A}_{i}+\widetilde{A}_{i}^{T} P+P \widetilde{U}_{i} \widetilde{F}_{i}(t) \widetilde{M}_{i}\right. \\
& \left.+\widetilde{M}_{i}^{T} \widetilde{F}_{i}^{T}(t) \widetilde{U}_{i}^{T} P\right] \xi(t) \\
& \leqslant \sum_{i=1}^{r} h_{i}(\theta(t)) \xi^{T}(t)\left[P \widetilde{A}_{i}+\widetilde{A}_{i}^{T} P+\mu^{-1} P \widetilde{U}_{i} \widetilde{U}_{i}^{T} P\right. \\
& \left.+\mu \widetilde{M}_{i}^{T} \widetilde{M}_{i}\right] \xi(t) .
\end{aligned}
$$

Inequality (14) implies that $P \widetilde{A}_{i}+\widetilde{A}_{i}^{T} P+\mu^{-1} P \widetilde{U}_{i} \widetilde{U}_{i}^{T} P+$ $\mu \widetilde{M}_{i}^{T} \widetilde{M}_{i}<0$ by Schur complement, and thus $\dot{V}(\xi(t), t)<0$. This implies that the error system in (8) with $u(t)=0$ is asymptotically stable.

Now, we will establish the $\mathscr{L}_{2}-\mathscr{L}_{\infty}$ performance for the error system in (8). Assume zero initial condition, we have

$$
\begin{aligned}
& \dot{V}(\xi(t), t)-u^{T}(t) u(t) \\
& =\sum_{i=1}^{r} h_{i}(\theta(t)) \xi^{T}(t)\left\{P\left[\widetilde{A}_{i}+\Delta \widetilde{A}_{i}(t)\right]+\left[\widetilde{A}_{i}+\Delta \widetilde{A}_{i}(t)\right]^{T} P\right\} \xi(t) \\
& \quad+2 \sum_{i=1}^{r} h_{i}(\theta(t)) \xi^{T}(t) P\left[\widetilde{B}_{i}+\Delta \widetilde{B}_{i}(t)\right] u(t)-u^{T}(t) u(t) \\
& \leqslant \sum_{i=1}^{r} h_{i}(\theta(t)) \xi^{T}(t)\left\{P \widetilde{A}_{i}+\widetilde{A}_{i}^{T} P+2 \mu^{-1} P \widetilde{U}_{i} \widetilde{U}_{i}^{T} P\right. \\
& \left.\quad+\mu \widetilde{M}_{i}^{T} \widetilde{M}_{i}\right\} \xi(t) \\
& \quad+2 \sum_{i=1}^{r} h_{i}(\theta(t)) \xi^{T}(t) P \widetilde{B}_{i} u(t)+\mu u^{T}(t) \widetilde{N}_{i}^{T} \widetilde{N}_{i} u(t) \\
& \quad-u^{T}(t) u(t) \\
& =\sum_{i=1}^{r} h_{i}(\theta(t))\left[\begin{array}{l}
\xi(t) \\
u(t)
\end{array}\right]^{T}\left[\begin{array}{c}
\Upsilon_{11 i} \\
\star
\end{array} \begin{array}{l}
\Upsilon_{22 i} \\
\Upsilon_{12 i} \\
u(t)
\end{array}\right],
\end{aligned}
$$

where

$$
\Upsilon_{11 i} \triangleq P \widetilde{A}_{i}+\widetilde{A}_{i}^{T} P+2 \mu^{-1} P \widetilde{U}_{i} \widetilde{U}_{i}^{T} P+\mu \widetilde{M}_{i}^{T} \widetilde{M}_{i}
$$

$$
\Upsilon_{12 i} \triangleq P \widetilde{B}_{i}, \quad \Upsilon_{22 i} \triangleq \mu \widetilde{N}_{i}^{T} \widetilde{N}_{i}-I
$$

Considering (14) and (19), we have

$$
\dot{V}(\xi(t), t)-u^{T}(t) u(t)<0
$$

Thus, integrating both sides of (21) from zero to $t^{\star}$, we get

$$
\int_{0}^{t^{\star}} \dot{V}(\xi(t), t) d t<\int_{0}^{t^{\star}} u^{T}(t) u(t) d t<\int_{0}^{\infty} u^{T}(t) u(t) d t
$$

Considering the zero initial condition and (16), we have

$$
\xi^{T}\left(t^{\star}\right) P \xi\left(t^{\star}\right)<\int_{0}^{\infty} u^{T}(t) u(t) d t
$$

Then, based on (15) and Schur complement, we have

$$
e^{T}(t) e(t) \leq \gamma^{2} \xi^{T}(t) P \xi(t)
$$

Combining (23) and (24) together, we have

$$
\sup _{\forall t}\left[e^{T}(t) e(t)\right]<\gamma^{2} \int_{0}^{\infty} u^{T}(t) u(t) d t
$$

The proof is completed.

Based on the obtained result in Theorem 3, we will present the method to solve the $\mathscr{L}_{2}-\mathscr{L}_{\infty}$ model approximation for the T-S fuzzy uncertain system in (4) by the convex linearization procedure.

\section{4. $\mathscr{L}_{2}-\mathscr{L}_{\infty}$ Model Reduction}

In this section, we are ready to give a solution to the $\mathscr{L}_{2}-\mathscr{L}_{\infty}$ model reduction for the T-S fuzzy uncertain system in (4).

Theorem 4. Consider the continuous-time T-S fuzzy uncertain system in (4). There exists a reduced-order uncertain model in the form of (6) that solves the $\mathscr{L}_{2}-\mathscr{L}_{\infty}$ model reduction problem with (13) satisfied, if there exist matrices 
$\mathscr{P}>0, \mathbb{Q}>0, \mathscr{A}, \mathscr{B}, \mathscr{C}, \mathscr{D}, \mathscr{U}, \mathscr{M}$, and $\mathcal{N}$ such that for $i=1,2, \ldots, r$,

$$
\left[\begin{array}{ccccccccc}
\Pi_{11 i} & \Pi_{12 i} & \Pi_{13 i} & \mathscr{P U}_{i} & \mathscr{H} \mathcal{U} & M_{i}^{T} & 0 & 0 & 0 \\
\star & \Pi_{22} & \Pi_{23 i} & \mathcal{Q}^{T} U_{i} & \mathscr{U}^{T} & 0 & \mathscr{M}^{T} & 0 & 0 \\
\star & \star & -I & 0 & 0 & 0 & 0 & N_{i}^{T} & \mathcal{N}^{T} \\
\star & \star & \star & -\frac{\mu}{2} I & 0 & 0 & 0 & 0 & 0 \\
\star & \star & \star & \star & -\frac{\mu}{2} I & 0 & 0 & 0 & 0 \\
\star & \star & \star & \star & \star & -\frac{1}{\mu} I & 0 & 0 & 0 \\
\star & \star & \star & \star & \star & \star & -\frac{1}{\mu} I & 0 & 0 \\
\star & \star & \star & \star & \star & \star & \star & -\frac{1}{\mu} I & 0 \\
\star & \star & \star & \star & \star & \star & \star & \star & -\frac{1}{\mu} I \\
& & & & & & & &
\end{array}\right]
$$

$<0$,

$$
\left[\begin{array}{ccc}
\Xi_{11 i} & \Xi_{12 i} & \Xi_{13 i} \\
\star & \Xi_{22 i} & \Xi_{23 i} \\
\star & \star & \Xi_{33 i}
\end{array}\right] \geq 0
$$

where

$$
\begin{gathered}
\mathscr{H} \triangleq\left[\begin{array}{c}
I_{k \times k} \\
0_{(n-k) \times k}
\end{array}\right], \\
\Pi_{11 i} \triangleq \mathscr{P} A_{i}+A_{i}^{T} \mathscr{P}, \quad \Pi_{12 i} \triangleq \mathscr{H} \mathscr{A}+A_{i}^{T} \mathscr{H} Q \\
\Pi_{22} \triangleq \mathscr{A}+\mathscr{A}^{T}, \\
\Pi_{13 i} \triangleq \mathscr{P} B_{i}+\mathscr{H} \mathscr{B}, \quad \Pi_{23 i} \triangleq Q \mathscr{H}^{T} B_{i}+\mathscr{B}, \\
\Xi_{33 i} \triangleq \gamma^{2} I, \\
\Xi_{11 i} \triangleq \mathscr{P}, \quad \Xi_{12 i} \triangleq \mathscr{H} Q, \quad \Xi_{13 i} \triangleq C_{i}^{T}, \\
\Xi_{22 i} \triangleq \mathscr{Q}, \quad \Xi_{23 i} \triangleq-\mathscr{C}^{T} .
\end{gathered}
$$

Moreover, if the above conditions are feasible, then the system matrices of an admissible reduced-order model in the form of (6) can be calculated from

$$
\left[\begin{array}{cc|c}
\widehat{A} & \widehat{B} & \widehat{U} \\
\widehat{C} & \widehat{D} & 0 \\
\hline \widehat{M} & 0 & \widehat{N}
\end{array}\right]=\left[\begin{array}{cc|c}
\mathscr{Q}^{-1} & 0 & 0 \\
0 & I & 0 \\
\hline 0 & 0 & I
\end{array}\right]\left[\begin{array}{cc|c}
\mathscr{A} & \mathscr{B} & \mathscr{U} \\
\mathscr{C} & \mathscr{D} & 0 \\
\hline \mathscr{M} & 0 & \mathcal{N}
\end{array}\right] .
$$

Proof. According to Theorem 3, $P$ is nonsingular since $P>0$. Now, partition $P$ as

$$
P \triangleq\left[\begin{array}{c|c}
P_{1} & P_{2} \\
\hline \star & P_{3}
\end{array}\right], \quad P_{2} \triangleq\left[\begin{array}{c}
P_{4} \\
\hline 0_{(n-k) \times k}
\end{array}\right],
$$

where $P_{1} \in \mathbb{R}^{n \times n}$ and $P_{3} \in \mathbb{R}^{k \times k}$ are symmetric positive definite matrices, $P_{2} \in \mathbb{R}^{n \times k}$, and $P_{4} \in \mathbb{R}^{k \times k}$. Without loss of generality, we assume that $P_{4}$ is nonsingular. To see this, let the matrix $S \triangleq P+\alpha T$, where $\alpha$ is a positive scalar and

$$
\begin{gathered}
T \triangleq\left[\begin{array}{c|c}
0_{n \times n} & \mathscr{H} \\
\hline \star & 0_{k \times k}
\end{array}\right], \quad S \triangleq\left[\begin{array}{c|c}
S_{1} & S_{2} \\
\hline \star & S_{3}
\end{array}\right], \\
S_{2} \triangleq\left[\begin{array}{c}
S_{4} \\
\hline 0_{(n-k) \times k}
\end{array}\right] .
\end{gathered}
$$

Observe that since $P>0$, we have that $S>0$ for $\alpha>0$ in the neighborhood of the origin. Thus, it can be easily verified that there exists an arbitrarily small $\alpha>0$ such that $S_{4}$ is nonsingular and (14)-(15) are feasible with $P$ replaced by $S$. Since $S_{4}$ is nonsingular, we thus conclude that there is no loss of generality to assume the matrix $P_{4}$ to be nonsingular.

Define the following matrices which are also nonsingular:

$$
\mathscr{J} \triangleq\left[\begin{array}{c|c}
I & 0 \\
\hline 0 & P_{3}^{-1} P_{4}^{T}
\end{array}\right], \quad \mathscr{P} \triangleq P_{1}, \quad Q \triangleq P_{4} P_{3}^{-1} P_{4}^{T},
$$

$$
\begin{aligned}
{\left[\begin{array}{cc|c}
\mathscr{A} & \mathscr{B} & \mathcal{U} \\
\mathscr{C} & \mathscr{D} & 0 \\
\hline \mathscr{M} & 0 & \mathcal{N}
\end{array}\right] \triangleq } & {\left[\begin{array}{cc|c}
P_{4} & 0 & 0 \\
0 & I & 0 \\
\hline 0 & 0 & I
\end{array}\right]\left[\begin{array}{cc|c}
\widehat{A} & \widehat{B} & \widehat{U} \\
\widehat{C} & \widehat{D} & 0 \\
\hline \widehat{M} & 0 & \widehat{N}
\end{array}\right] } \\
& \times\left[\begin{array}{cc|c}
P_{3}^{-1} P_{4}^{T} & 0 & 0 \\
0 & I & 0 \\
\hline 0 & 0 & I
\end{array}\right] .
\end{aligned}
$$

Performing a congruence transformation to (14) and (15) by $\operatorname{diag}(\mathscr{J}, I)$ and $\operatorname{diag}(\mathscr{J}, I, I)$, respectively, we obtain

$$
\begin{aligned}
& {\left[\begin{array}{ccccc}
\mathscr{J}^{T}\left(P \widetilde{A}_{i}+\widetilde{A}_{i}^{T} P\right) \mathcal{J} & \mathscr{J}^{T} P \widetilde{B}_{i} & \mathscr{J}^{T} P \widetilde{U}_{i} & \mathscr{J}^{T} \widetilde{M}_{i}^{T} & 0 \\
\star & -I & 0 & 0 & \widetilde{N}_{i}^{T} \\
\star & \star & -\frac{\mu}{2} I & 0 & 0 \\
\star & \star & \star & -\frac{1}{\mu} I & 0 \\
\star & \star & \star & \star & -\frac{1}{\mu} I
\end{array}\right]<0,} \\
& {\left[\begin{array}{cc}
\mathscr{J}^{T} P \mathscr{J} & \mathscr{J}^{T} \widetilde{C}_{i}^{T} \\
\star & \gamma^{2} I
\end{array}\right] \geq 0,}
\end{aligned}
$$

where

$$
\begin{gathered}
\mathscr{J}^{T} P \widetilde{A}_{i} \mathscr{J} \triangleq\left[\begin{array}{cc}
\mathscr{P} A_{i} & \mathscr{H} \mathscr{A} \\
\mathcal{Q} \mathscr{H}^{T} A_{i} & \mathscr{A}
\end{array}\right], \\
\mathscr{J}^{T} P \widetilde{U}_{i} \triangleq\left[\begin{array}{cc}
\mathscr{P} U_{i} & \mathscr{H} \mathscr{U} \\
\mathcal{Q} \mathscr{H}^{T} U_{i} & \mathcal{U}
\end{array}\right], \\
\mathscr{J}^{T} P \widetilde{B}_{i} \triangleq\left[\begin{array}{c}
\mathscr{P} B_{i}+\mathscr{H} \mathscr{B} \\
\mathcal{Q} \mathscr{H}^{T} B_{i}+\mathscr{B}
\end{array}\right], \quad \mathscr{J}^{T} \widetilde{M}^{T} \triangleq\left[\begin{array}{cc}
M_{i}^{T} & 0 \\
0 & \mathscr{M}^{T}
\end{array}\right], \\
\mathscr{J}^{T} P \mathscr{J} \triangleq\left[\begin{array}{cc}
\mathscr{P} & \mathscr{H} Q \\
\star & \mathbb{Q}
\end{array}\right], \quad \widetilde{N}_{i} \triangleq\left[\begin{array}{c}
N_{i} \\
\mathscr{N}
\end{array}\right], \\
\widetilde{C}_{i} \mathscr{J} \triangleq\left[\begin{array}{ll}
C_{i} & -\mathscr{C}] .
\end{array}\right.
\end{gathered}
$$


Considering (36), we can obtain (26)-(27) from (34)-(35), respectively. Moreover, notice that (33) is equivalent to

$$
\begin{aligned}
{\left[\begin{array}{cc|c}
\widehat{A} & \widehat{B} & \widehat{U} \\
\widehat{C} & 0 & 0 \\
\hline \widehat{M} & 0 & \widehat{N}
\end{array}\right] \triangleq } & {\left[\begin{array}{cc|c}
P_{4}^{-1} & 0 & 0 \\
0 & I & 0 \\
\hline 0 & 0 & I
\end{array}\right]\left[\begin{array}{cc|c}
\mathscr{A} & \mathscr{B} & \mathcal{U} \\
\mathscr{C} & 0 & 0 \\
\hline \mathscr{M} & 0 & \mathcal{N}
\end{array}\right] } \\
& \times\left[\begin{array}{ccc|c}
P_{4}^{-T} P_{3} & 0 & 0 \\
0 & I & 0 \\
\hline 0 & 0 & I
\end{array}\right] \\
= & {\left[\begin{array}{cc|c|c|c}
\left(P_{4}^{-T} P_{3}\right)^{-1} & Q^{-1} & 0 & 0 \\
0 & & I & 0 \\
\hline 0 & & 0 & I
\end{array}\right]\left[\begin{array}{cc|c}
\mathscr{A} & \mathscr{B} & \mathscr{U} \\
\mathscr{C} & 0 & 0 \\
\hline \mathscr{M} & 0 & \mathcal{N}
\end{array}\right] } \\
& \times\left[\begin{array}{cc|c}
P_{4}^{-T} P_{3} & 0 & 0 \\
0 & I & 0 \\
\hline 0 & 0 & I
\end{array}\right] .
\end{aligned}
$$

Notice also that the matrices $\widehat{A}, \widehat{B}, \widehat{C}, \widehat{U}, \widehat{M}$, and $\widehat{N}$ in (6) can be written as (37), which implies that $P_{4}^{-T} P_{3}$ can be viewed as a similarity transformation on the state-space realization of the filter and, as such, has no effect on the filter mapping from $u$ to $\hat{y}$. Without loss of generality, we may set $P_{4}^{-T} P_{3}=$ $I$, thus obtaining (29). Therefore, the filter $(\widehat{\Sigma})$ in $(6)$ can be constructed by (29). This completes the proof.

Remark 5. Notice that the obtained conditions in Theorem 4 are all in linear matrix inequalities form; the fuzzy-ruleindependent $\mathscr{L}_{2}-\mathscr{L}_{\infty}$ model simplification can be determined by solving the following convex optimization problem:

$$
\min _{\mathscr{P}>0, \mathscr{Q}>0, \mathscr{A}, \mathscr{B}, \mathscr{C}, \mathscr{D}, \mathscr{U}, \mathscr{M}, \mathcal{N}} \delta
$$

$$
\text { subject to (26)-(27) (where } \left.\delta=\gamma^{2}\right) \text {. }
$$

\section{Illustrative Example}

Consider the continuous-time nonlinear uncertain system. The T-S fuzzy model of this nonlinear uncertain system is presented as follows.

\subsection{Plant Form}

Rule 1. IF $x_{1}(t)$ is $\mu_{1}$, then

$$
\begin{gathered}
\dot{x}(t)=\left[A_{1}+\Delta A_{1}(t)\right] x(t)+\left[B_{1}+\Delta B_{1}(t)\right] u(t), \\
y(t)=C_{1} x(t) .
\end{gathered}
$$

Rule 2. IF $x_{1}(t)$ is $\mu_{2}$, then

$$
\begin{gathered}
\dot{x}(t)=\left[A_{2}+\Delta A_{2}(t)\right] x(t)+\left[B_{2}+\Delta B_{2}(t)\right] u(t), \\
y(t)=C_{2} x(t),
\end{gathered}
$$

where

$$
\begin{aligned}
& \Delta A_{1}(t) \\
& =\left[\begin{array}{cccc}
-0.02 \sin (t) & 0.03 \sin (t) & -0.04 \sin (t) & 0.02 \sin (t) \\
0.04 \sin (t) & -0.06 \sin (t) & 0.08 \sin (t) & -0.04 \sin (t) \\
0.02 \sin (t) & -0.03 \sin (t) & 0.04 \sin (t) & -0.02 \sin (t) \\
-0.06 \sin (t) & 0.09 \sin (t) & -0.12 \sin (t) & 0.06 \sin (t)
\end{array}\right], \\
& \Delta A_{2}(t) \\
& =\left[\begin{array}{cccc}
0.02 \sin (t) & -0.02 \sin (t) & 0.04 \sin (t) & -0.06 \sin (t) \\
-0.01 \sin (t) & 0.01 \sin (t) & -0.02 \sin (t) & 0.03 \sin (t) \\
0.02 \sin (t) & -0.02 \sin (t) & 0.04 \sin (t) & -0.06 \sin (t) \\
0.04 \sin (t) & -0.04 \sin (t) & 0.08 \sin (t) & -0.12 \sin (t)
\end{array}\right], \\
& \Delta B_{1}(t)=\left[\begin{array}{c}
0.05 \sin (t) \\
-0.10 \sin (t) \\
-0.05 \sin (t) \\
0.15 \sin (t)
\end{array}\right], \quad \Delta B_{2}(t)=\left[\begin{array}{c}
-0.04 \sin (t) \\
0.02 \sin (t) \\
-0.04 \sin (t) \\
-0.08 \sin (t)
\end{array}\right] \text {, } \\
& A_{1}=\left[\begin{array}{cccc}
-2.2 & 0.3 & 0.2 & 0.1 \\
0.2 & -4.2 & 0.2 & 0.1 \\
0.3 & 0 & -3.1 & 0.2 \\
0.4 & 0.2 & 0.1 & -1.5
\end{array}\right], \quad B_{1}=\left[\begin{array}{c}
2.4 \\
1 \\
1.3 \\
2
\end{array}\right] \text {, } \\
& A_{2}=\left[\begin{array}{cccc}
-2.5 & 0.1 & 0.3 & 0.2 \\
0.2 & -4.1 & 0.1 & 0.2 \\
0.1 & 0 & -2.9 & 0.2 \\
0.4 & 0.2 & 0.1 & -1.7
\end{array}\right], \quad B_{2}=\left[\begin{array}{l}
2.2 \\
1.1 \\
1.2 \\
2.1
\end{array}\right] \text {, } \\
& C_{1}=\left[\begin{array}{llll}
1 & 1.1 & 0.6 & 0.3
\end{array}\right], \quad C_{2}=\left[\begin{array}{llll}
1.1 & 1 & 0.5 & 0.3
\end{array}\right] \text {. }
\end{aligned}
$$

Here, we are interested in finding reduced-order systems (Case 1: $k=3$; Case 2: $k=2$; Case 3: $k=1$ ) in the form of $(\widehat{\Sigma})$ in $(6)$ to approximate the above system in an $\mathscr{L}_{2}-\mathscr{L}_{\infty}$ sense by using the convex linearization. Solving the (26)-(29) in Theorem 4, we have the results for different cases shown as follows.

Case 1. with $k=3$, the minimum $\gamma$ is $\gamma^{*}=0.5367$ and

$$
\begin{gathered}
\mathscr{P}=\left[\begin{array}{cccc}
80.5202 & 78.7268 & -79.2386 & -2.3421 \\
78.7268 & 116.0219 & -75.9371 & -0.4125 \\
-79.2386 & -75.9371 & 93.7412 & 3.0132 \\
-2.3421 & -0.4125 & 3.0132 & 0.8390
\end{array}\right], \\
\mathscr{Q}=\left[\begin{array}{cccc}
66.0499 & 77.0315 & -54.9810 \\
77.0315 & 114.8214 & -72.8165 \\
-54.9810 & -72.8165 & 50.6632
\end{array}\right],
\end{gathered}
$$

$$
\begin{aligned}
& {\left[\begin{array}{cc|c}
\widehat{A} & \widehat{B} & \widehat{U} \\
\widehat{C} & 0 & 0 \\
\hline \widehat{M} & 0 & \widehat{N}
\end{array}\right]} \\
& =\left[\begin{array}{cccc|c}
-0.9192 & 1.7384 & -3.0426 & -2.2476 & -0.0127 \\
1.1148 & -2.4321 & -2.3040 & -0.9573 & -0.0363 \\
2.9105 & 4.0517 & -10.1880 & -1.0748 & -0.0679 \\
-2.2244 & -1.2487 & 1.2255 & 0.0000 & 0.0000 \\
\hline-0.0334 & 0.0501 & -0.0667 & 0.0000 & 0.0834
\end{array}\right] .
\end{aligned}
$$


TABLE 1: Achieved $\gamma^{*}$ for the obtained reduced-order models.

\begin{tabular}{lccc}
\hline Reduced-order model & $k=3$ & $k=2$ & $k=1$ \\
\hline $\begin{array}{l}\text { Optimal } \mathscr{L}_{2}-\mathscr{L}_{\infty} \\
\text { performance }\end{array}$ & $\gamma^{*}=0.5367$ & $\gamma^{*}=0.5459$ & $\gamma^{*}=0.6652$ \\
\hline
\end{tabular}

Case 2. with $k=2$, the minimum $\gamma$ is $\gamma^{*}=0.5459$ and

$$
\begin{gathered}
\mathscr{P}=\left[\begin{array}{cccc}
23.1397 & 14.2678 & -10.3622 & -2.1777 \\
14.2678 & 41.5106 & 1.5331 & -0.0137 \\
-10.3622 & 1.5331 & 14.6263 & 2.0607 \\
-2.1777 & -0.0137 & 2.0607 & 0.9969
\end{array}\right], \quad(44) \\
\mathscr{Q}=\left[\begin{array}{ccc}
14.9535 & 15.2591 \\
15.2591 & 40.7583
\end{array}\right], \\
{\left[\begin{array}{cc|c}
\widehat{A} & \widehat{B} & \widehat{U} \\
\widehat{C} & 0 & 0 \\
\hline \widehat{M} & 0 & \widehat{N}
\end{array}\right]=\left[\begin{array}{ccc|c}
-2.0175 & 0.2412 & -2.2984 & 0.0189 \\
0.4053 & -3.9904 & -1.0161 & -0.0120 \\
-1.6650 & -1.0603 & 0.0000 & 0.0000 \\
\hline-0.0216 & 0.0325 & 0.0000 & 0.0541
\end{array}\right] .}
\end{gathered}
$$

Case 3. with $k=1$, the minimum $\gamma$ is $\gamma^{*}=0.6652$ and

$$
\begin{gathered}
\mathscr{P}=\left[\begin{array}{cccc}
26.6722 & -7.4024 & -14.8699 & -2.4648 \\
-7.4024 & 8.5939 & 4.5852 & 1.8838 \\
-14.8699 & 4.5852 & 24.6985 & 1.1307 \\
-2.4648 & 1.8838 & 1.1307 & 1.0156
\end{array}\right], \\
\mathscr{Q}=\left[\begin{array}{cc}
13.8424
\end{array}\right], \\
{\left[\begin{array}{cc|c}
\widehat{A} & \widehat{B} & \widehat{U} \\
\widehat{C} & 0 & 0 \\
\hline \widehat{M} & 0 & \widehat{N}
\end{array}\right]=\left[\begin{array}{cc|c}
-2.0108 & -2.3112 & 0.0072 \\
-2.0181 & 0.0000 & 0.0000 \\
\hline-0.0144 & 0.0000 & 0.0361
\end{array}\right] .}
\end{gathered}
$$

In Table 1 , the achieved $\gamma^{*}$ for different cases can be arranged by solving the convex optimization problem, as discussed in Remark 5.

In addition, to show the model reduction performances of the obtained reduced-order systems, let the initial condition be zero, that is, $\widetilde{x}(0)=0(x(0)=0, \widehat{x}(0)=0)$, and suppose the membership functions to be

$$
\begin{gathered}
h_{1}\left(x_{1}(t)\right) \triangleq \exp \left[-\frac{x_{1}(t)-\vartheta}{2 \sigma^{2}}\right], \\
h_{2}\left(x_{1}(t)\right) \triangleq 1-\exp \left[-\frac{x_{1}(t)-\vartheta}{2 \sigma^{2}}\right],
\end{gathered}
$$

which are shown in Figure 1, and the exogenous input $u(t)$ is

$$
u(t)=\exp (-0.7 t) \sin (2 t), \quad t \geq 0 .
$$

Figure 2 shows the outputs of the original system (39)-(40) (black line), the third-order reduced model (43) (red line), the second-order reduced model (45) (blue line), and the firstorder reduced model (47) (green line) due to the above input signal. The output errors between the original system and the reduced models are shown in Figure 3.

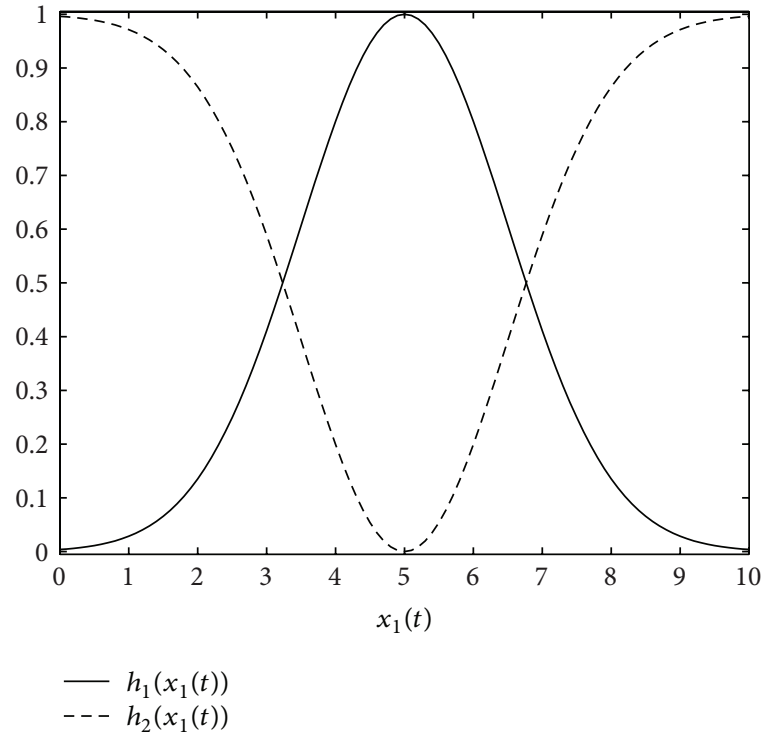

FIGURE 1: Membership functions for the two-fuzzy set for Example 1 .

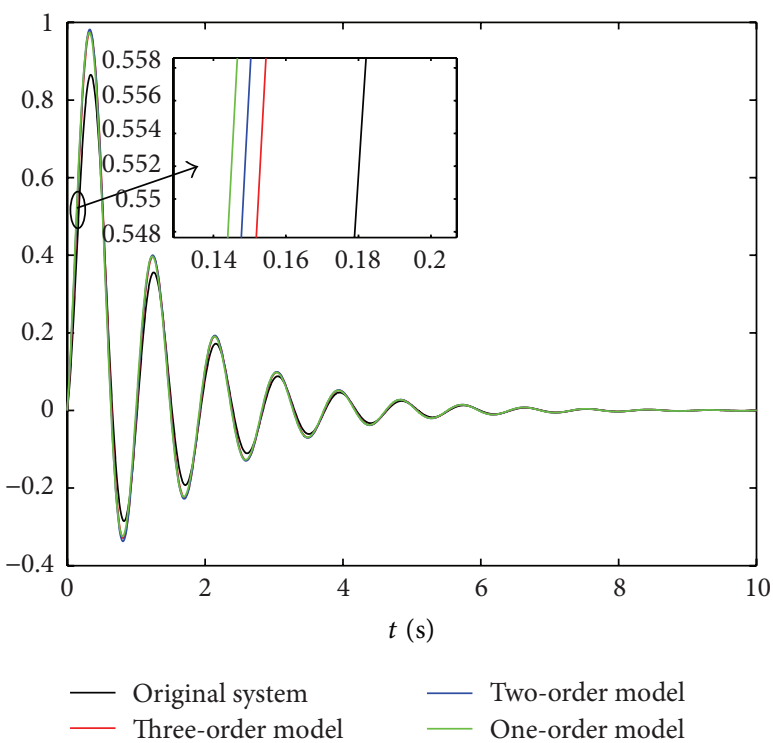

FIGURE 2: Outputs of the original system and the reduced-order models.

\section{Conclusions}

In this paper, the $\mathscr{L}_{2}-\mathscr{L}_{\infty}$ model reduction problem has been solved for T-S fuzzy uncertain systems by employing the convex linearization approach. Sufficient conditions of T-S fuzzy uncertain system have been proposed for the asymptotic stability with an $\mathscr{L}_{2}-\mathscr{L}_{\text {o }}$ performance of the approximation error. The corresponding solvability conditions for the reduced-order models by using the convex linearization approach have been derived. The presented numerical example has shown the utility of the proposed model approximation method. This paper talks about the model reduction problem for nonlinear systems by 


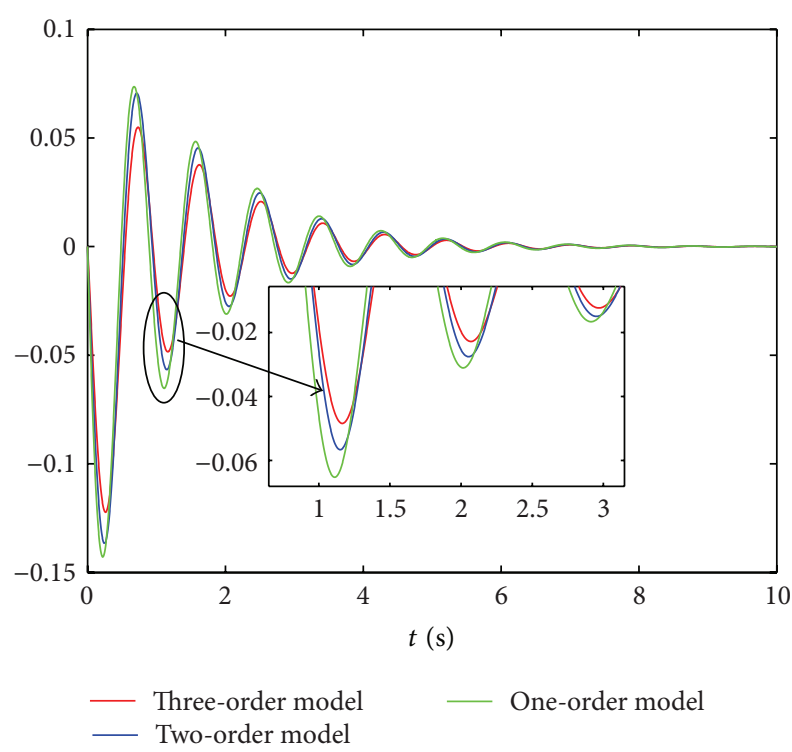

FIGURE 3: Output errors between the original system and the reduced-order models.

employing the convex linearization approach. In order to achieve more practical oriented results, further work could be considered under data-driven (measurements) framework $[40,41]$. The future topics, for examlpe, control [42] and fault tolerant scheme [43] in the identical framework seem more interesting from both academic and industrial domains.

\section{Conflict of Interests}

The authors declare that there is no conflict of interests regarding the publication of this paper.

\section{Acknowledgment}

This work was partly supported in part by the National Natural Science Foundation of China under Grant (61203035) and by the Polish-Norwegian Research Programme operated by the National Centre for Research and 24 Development under the Norwegian Financial Mechanism 2009-2014 in the frame of Project Contract No. Pol-Nor/200957/47/2013.

\section{References}

[1] T. Takagi and M. Sugeno, "Fuzzy identification of systems and its applications to modeling and control," IEEE Transactions on Systems, Man and Cybernetics, vol. 15, no. 1, pp. 116-132, 1985.

[2] H. Gao, X. Liu, and J. Lam, "Stability analysis and stabilization for discrete-time fuzzy systems with time-varying delay," IEEE Transactions on Systems, Man, and Cybernetics B: Cybernetics, vol. 39, no. 2, pp. 306-317, 2009.

[3] H. Li, J. Yu, C. Hilton, and H. Liu, "Adaptive sliding mode control for nonlinear active suspension systems using T-S fuzzy model," IEEE Transactions on Industrial Electronics, vol. 60, no. 8, pp. 3328-3338, 2013.
[4] Y. Zhao, H. Gao, J. Lam, and B. Du, "Stability and stabilization of delayed T-S fuzzy systems: a delay partitioning approach," IEEE Transactions on Fuzzy Systems, vol. 17, no. 4, pp. 750-762, 2009.

[5] X. Zhao, L. Zhang, P. Shi, and H. R. Karimi, "Novel stability criteria for T-S fuzzy systems," IEEE Transactions on Fuzzy Systems, vol. 22, no. 2, pp. 313-323, 2014.

[6] Z. Wang, D. W. C. Ho, and X. Liu, "A note on the robust stability of uncertain stochastic fuzzy systems with time-delays," IEEE Transactions on Systems, Man, and Cybernetics A: Systems and Humans, vol. 34, no. 4, pp. 570-576, 2004.

[7] H. Zhang, X. Zhang, and J. Wang, "Robust gain-scheduling energy-to-peak control of vehicle lateral dynamics stabilisation," Vehicle System Dynamics, vol. 52, no. 3, pp. 1-32, 2014.

[8] M. Chadli and H. R. Karimi, "Robust observer design for unknown inputs Takagi-Sugeno models," IEEE Transactions on Fuzzy Systems, vol. 21, no. 1, pp. 158-164, 2013.

[9] H. Li, H. Liu, H. Gao, and P. Shi, "Reliable fuzzy control for active suspension systems with actuator delay and fault," IEEE Transactions on Fuzzy Systems, vol. 20, no. 2, pp. 342-357, 2012.

[10] X. Su, P. Shi, L. Wu, and Y. D. Song, "A novel control design on discrete-time Takagi-Sugeno fuzzy systems with time-varying delays," IEEE Transactions on Fuzzy Systems, vol. 21, no. 4, pp. 655-671, 2013.

[11] H. Li, X. Jing, H. K. Lam, and P. Shi, "Fuzzy sampleddata control for uncertain vehicle suspension systems," IEEE Transactions on Cybernetics, no. 99, 2013.

[12] Y. Zhang, C. Liu, and H. R. Karimi, "Comments on 'Finite-time $\mathscr{H}_{\infty}$ fuzzy control of nonlinear jump systems with time delays via dynamic observer-based state feedback', IEEE Transactions on Fuzzy Systems, vol. 22, no. 1, pp. 230-233, 2014.

[13] T. Youssef, M. Chadli, H. R. Karimi, and M. Zelmat, "Design of unknown inputs proportional integral observers for T-S fuzzy models," Neurocomputing, vol. 123, pp. 156-165, 2014.

[14] L. Zhao, H. Gao, and H. R. Karimi, "Robust stability and stabilization of uncertain T-S fuzzy systems with time-varying delay: an input-output approach," IEEE Transactions on Fuzzy Systems, vol. 21, no. 5, pp. 883-897, 2013.

[15] H. Li, Y. Pan, and Q. Zhou, "Filter design for interval type2 fuzzy systems with D stability constraints under a unified frame," IEEE Transactions on Fuzzy Systems, no. 99, 2014.

[16] H. Dong, Z. Wang, D. W. C. Ho, and H. Gao, "Robust $\mathscr{H}_{\infty}$ fuzzy output-feedback control with multiple probabilistic delays and multiple missing measurements," IEEE Transactions on Fuzzy Systems, vol. 18, no. 4, pp. 712-725, 2010.

[17] H. Zhang, Y. Shi, and J. Wang, "Observer-based tracking controller design for networked predictive control systems with uncertain Markov delays," International Journal of Control, vol. 86 , no. 10, pp. 1824-1836, 2013.

[18] M. Chadli and T. M. Guerra, "LMI solution for robust static output feedback control of discrete Takagi-Sugeno fuzzy models," IEEE Transactions on Fuzzy Systems, vol. 20, no. 6, pp. 1160-1165, 2012.

[19] X. Su, L. Wu, and P. Shi, "Senor networks with random link failures: distributed filtering for T-S fuzzy systems," IEEE Transactions on Industrial Informatics, vol. 9, no. 3, pp. 17391750, 2013

[20] X. Su, P. Shi, L. Wu, and S. K. Nguang, "Induced $\mathscr{L}_{2}$ filtering of fuzzy stochastic systems with time-varying delays," IEEE Transactions on Cybernetics, vol. 43, no. 4, pp. 1251-1264, 2013. 
[21] H. Dong, Z. Wang, and H. Gao, "Robust $\mathscr{H}_{\infty}$ filtering for a class of nonlinear networked systems with multiple stochastic communication delays and packet dropouts," IEEE Transactions on Signal Processing, vol. 58, no. 4, pp. 1957-1966, 2010.

[22] Y. Zhao, J. Lam, and H. Gao, “On energy-to-peak filtering for nonuniformly sampled nonlinear systems: a Markovian jump system approach," IEEE Transactions on Fuzzy Systems, vol. 22, no. 1, pp. 212-222, 2013.

[23] H. Zhang, A. S. Mehr, and Y. Shi, "Improved robust energy-topeak filtering for uncertain linear systems," Signal Processing, vol. 90, no. 9, pp. 2667-2675, 2010.

[24] L. Wu and D. W. C. Ho, "Fuzzy filter design for Itô stochastic systems with application to sensor fault detection," IEEE Transactions on Fuzzy Systems, vol. 17, no. 1, pp. 233-242, 2009.

[25] Y. Zhao, J. Lam, and H. Gao, "Fault detection for fuzzy systems with intermittent measurements," IEEE Transactions on Fuzzy Systems, vol. 17, no. 2, pp. 398-410, 2009.

[26] H. Dong, Z. Wang, J. Lam, and H. Gao, "Fuzzy-model-based robust fault detection with stochastic mixed time delays and successive packet dropouts," IEEE Transactions on Systems, Man, and Cybernetics B: Cybernetics, vol. 42, no. 2, pp. 365-376, 2012.

[27] J. Lam, H. Gao, S. Xu, and C. Wang, " $H_{\infty}$ and $\mathscr{L}_{2}-\mathscr{L}_{\infty}$ model reduction for system input with sector nonlinearities," Journal of Optimization Theory and Applications, vol. 125, no. 1, pp. 137155, 2005.

[28] Q. Wang, J. Lam, S. Xu, and H. Gao, "Delay-dependent and delay-independent energy-to-peak model approximation for systems with time-varying delay," International Journal of Systems Science, vol. 36, no. 8, pp. 445-460, 2005.

[29] X. Su, L. Wu, P. Shi, and Y. D. Song, " $\mathscr{H}_{\infty}$ model reduction of Takagi-Sugeno fuzzy stochastic systems," IEEE Transactions on Systems, Man, and Cybernetics B: Cybernetics, vol. 42, no. 6, pp. 1574-1585, 2012.

[30] L. Wu and W. X. Zheng, "Weighted $\mathscr{H}_{\infty}$ model reduction for linear switched systems with time-varying delay," Automatica, vol. 45, no. 1, pp. 186-193, 2009.

[31] L. Wu, X. Su, P. Shi, and J. Qiu, "Model approximation for discrete-time state-delay systems in the TS fuzzy framework," IEEE Transactions on Fuzzy Systems, vol. 19, no. 2, pp. 366-378, 2011.

[32] S. Xu and T. Chen, " $\mathscr{H}_{\infty}$ model reduction in the stochastic framework," SIAM Journal on Control and Optimization, vol. 42, no. 4, pp. 1293-1309, 2003.

[33] W.-Y. Yan and J. Lam, "An approximate approach to $\mathscr{H}_{2}$ optimal model reduction," IEEE Transactions on Automatic Control, vol. 44, no. 7, pp. 1341-1358, 1999.

[34] L. Zhang and J. Lam, "On $\mathscr{H}_{2}$ model reduction of bilinear systems," Automatica, vol. 38, no. 2, pp. 205-216, 2002.

[35] H. Gao, J. Lam, C. Wang, and Q. Wang, "Hankel norm approximation of linear systems with time-varying delay: continuous and discrete cases," International Journal of Control, vol. 77, no. 17, pp. 1503-1520, 2004.

[36] S. Boyd, L. El Ghaoui, E. Feron, and V. Balakrishnan, Linear Matrix Inequalities in System and Control Theory, vol. 15 of SIAM Studies in Applied Mathematics, SIAM, Philadelphia, Pa, USA, 1994.
[37] P. Gahinet and P. Apkarian, "A linear matrix inequality approach to $\mathscr{H}_{\infty}$ control," International Journal of Robust and Nonlinear Control, vol. 4, no. 4, pp. 421-448, 1994.

[38] H. Zhang, Y. Shi, A. Saadat Mehr, and H. Huang, "Robust FIR equalization for time-varying communication channels with intermittent observations via an LMI approach," Signal Processing, vol. 91, no. 7, pp. 1651-1658, 2011.

[39] L. Zhang, B. Huang, and J. Lam, " $\mathscr{L}_{2}$ gain analysis for switched systems with continuous-time and discrete-time subsystems," International Journal of Control, vol. 78, no. 15, pp. 1198-1205, 2005.

[40] S. Yin, S. Ding, A. Haghani, H. Hao, and P. Zhang, "A comparison study of basic data-driven fault diagnosis and process monitoring methods on the benchmark Tennessee Eastman process," Journal of Process Control, vol. 22, no. 9, pp. 1567-1581, 2012.

[41] S. Yin, S. X. Ding, A. H. A. Sari, and H. Hao, "Data-driven monitoring for stochastic systems and its application on batch process," International Journal of Systems Science, vol. 44, no. 7, pp. 1366-1376, 2013.

[42] S. Yin, S. Ding, and H. Luo, "Real-time implementation of fault tolerant control system with performance optimization," IEEE Transactions on Industrial Electronics, vol. 61, no. 5, pp. $2402-$ 2411, 2013.

[43] S. Yin, X. Yang, and H. R. Karimi, "Data-driven adaptive observer for fault diagnosis," Mathematical Problems in Engineering, vol. 2012, Article ID 832836, 21 pages, 2012. 


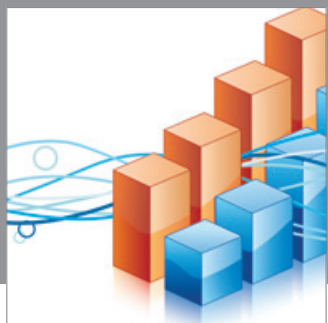

Advances in

Operations Research

mansans

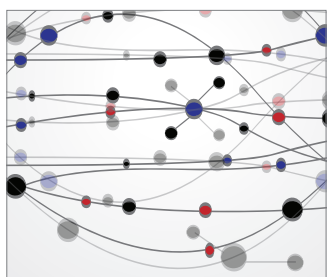

The Scientific World Journal
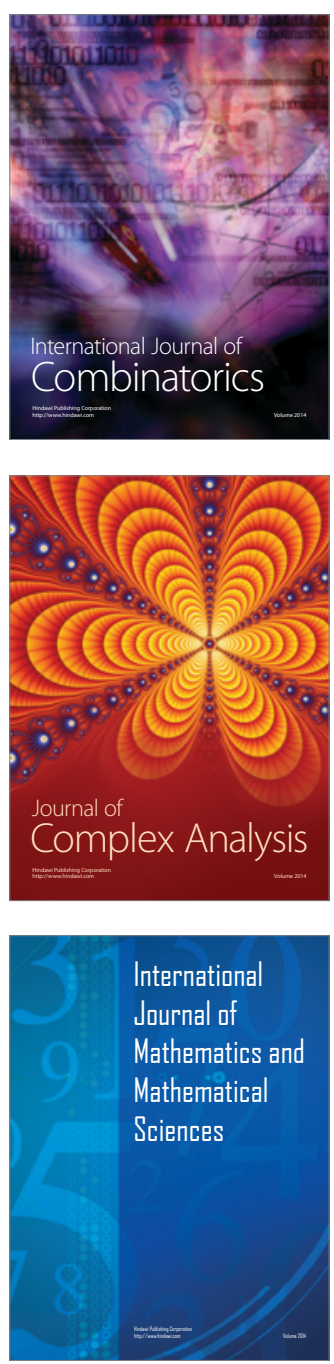
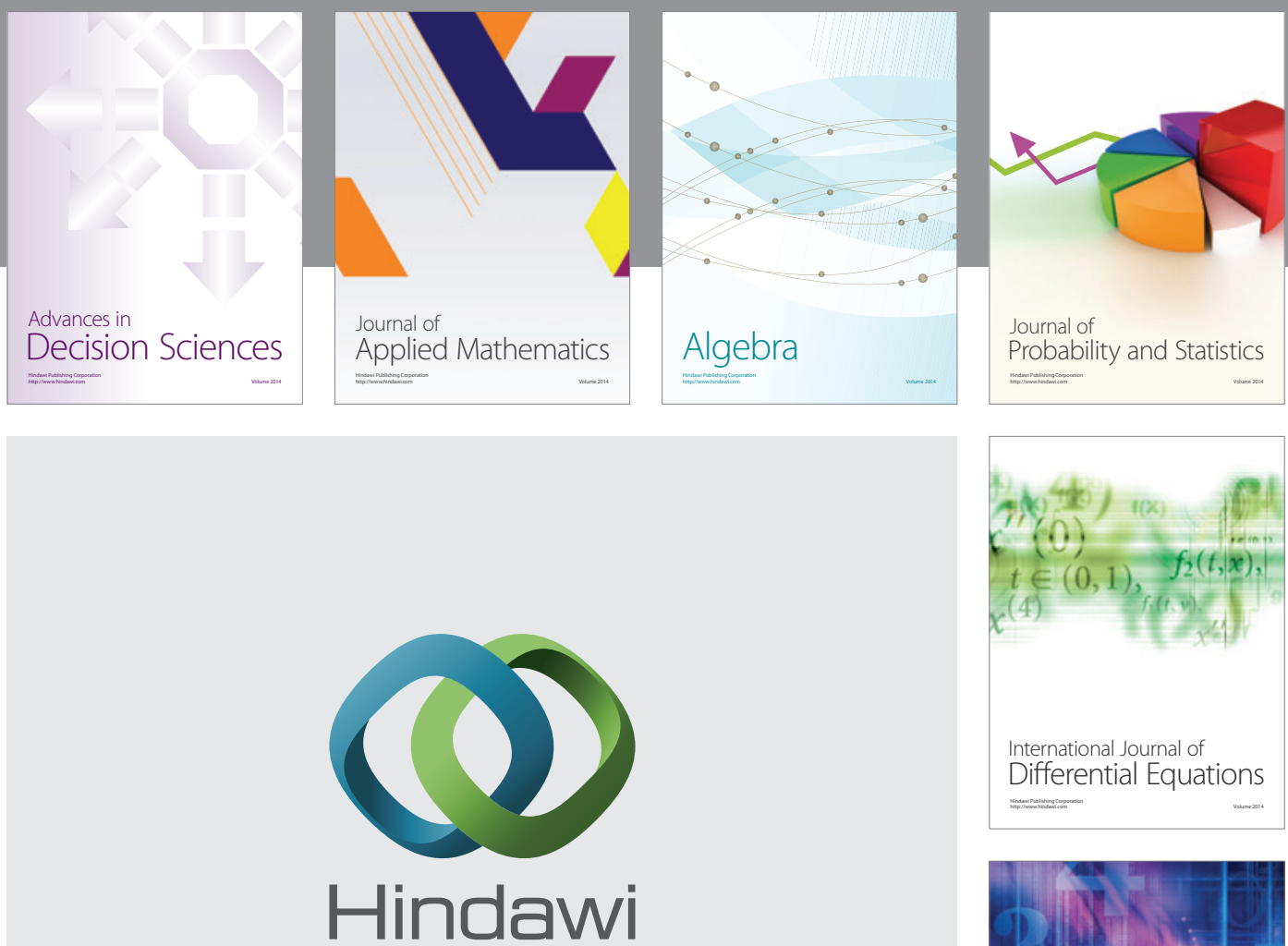

Submit your manuscripts at http://www.hindawi.com
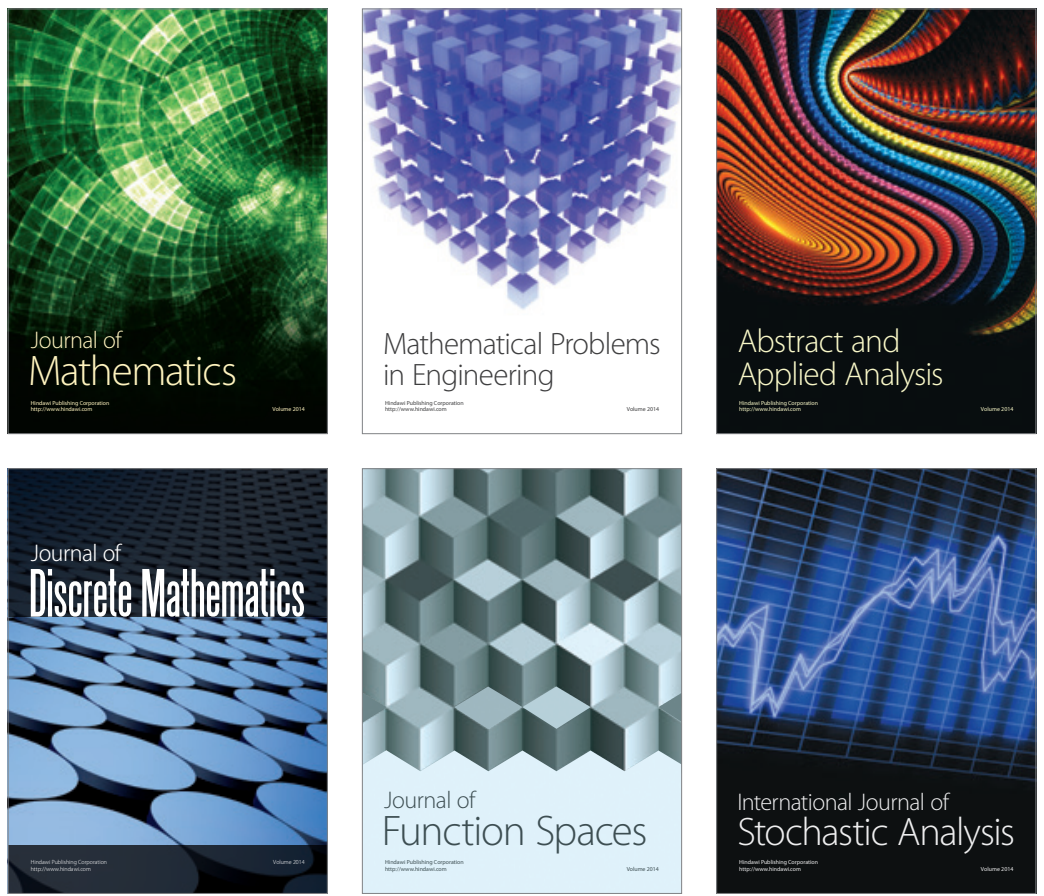

Journal of

Function Spaces

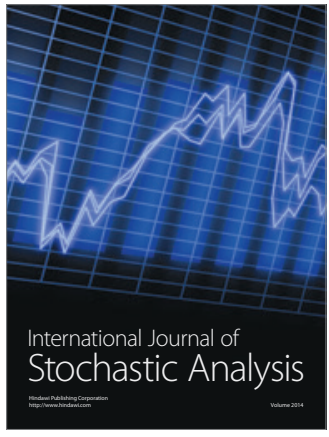

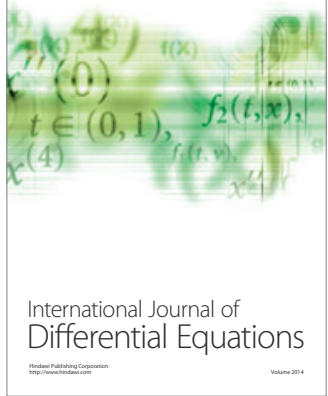
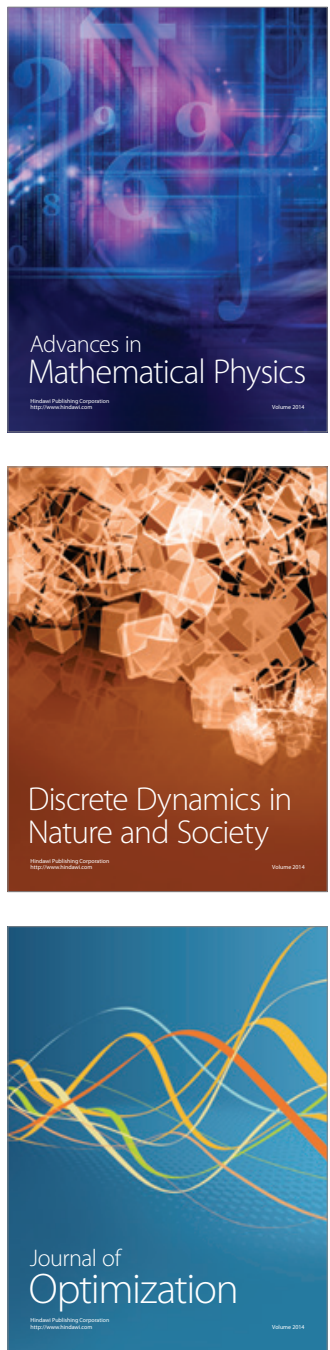